\title{
Research Paper: Prediction of Perceived Empathy Based on Emotional Schemas and Resilience in Mothers with Physically-Disabled Children
}

\author{
Dana Mohammad Aminzadeh ${ }^{1}$, ${ }^{*}$ Somaye Kazemian ${ }^{1}$, Masoumeh Esmaeily ${ }^{1}$, Yousef Asmari²
}

1. Department of Counseling, Faculty of Psychology and Educational Sciences, Allameh Tabataba'i University, Tehran, Iran.

2. Department of Educational Psychology, Faculty of Psychology and Educational Sciences, Allameh Tabataba'i University, Tehran, Iran.

\begin{tabular}{|c|c|}
\hline $\begin{array}{l}\text { Use vour device toscan ine } \\
\text { and read the article online }\end{array}$ & Citation: Mohammad Aminzadeh D. Kazemian S. Esmaeily M. Asmari Y. [Prediction of Perceived Empathy Based on \\
\hline 口偠要回 & $\begin{array}{l}\text { Emotional Schemas and Resilience in Mothers with Physically-Disabled Children (Persian)]. Archives of Rehabilitation. 2017; } \\
\text { 18(2):142-153. http://dx.doi.org/10.21859/jrehab-1802138 }\end{array}$ \\
\hline 回部 & doi : http://dx.doi.org/10.21859/jrehab-1802138 \\
\hline
\end{tabular}

Received: 31 Dec. 2016 Accepted: 08 Apr. 2017

Keywords:

Perceived empathy,

Emotional schemas, Resiliency, Mothers with disabled children

\section{ABSTRACT}

Objective Disability is a form of damage that limits one or more major life activities of an individual. It is also associated with psychological distress and depression in these individuals, which in turn also cause problems for the concerned families. Consequently, families with disabled children are socially isolated and experience a low-level of mental health and mood. As mothers of disabled children spend more time with them, their adjustment and physical and mental health are more threatened. They are more sensitive to emotional issues and family relationships outside the family. Thus, the present study was conducted to predict the perceived empathy based on emotional schemas and resilience in mothers with disabled children.

Materials \& Methods This study is a descriptive one, and the statistical population consisted of all mothers with motor-physically disabled children. This study was conducted in 2015-2016 academic year in the city of Tehran. Through purposeful sampling method, 120 samples were selected. Thus among the multiple areas in Tehran, areas 2, 4 and 16 were selected and by referring to special schools with physical-motor disabilities, questionnaires were given to the students. To collect information, Jefferson Scale of Patient's Perceptions of Physician Empathy Questionnaire (JSPPPE) was used to assess communication skills, human relations, quality, and expertise and medical skills. Connor-Davidson Resilience Scale was designed to measure the strength to cope with stress, and Emotional Schema Scale Lee Hee (LESS) that is used to determine beliefs and attitudes toward emotion in individuals were developed in 2002 by Lee Hee. Based on emotional schema, these two scales were used to measure 14 schema dimensions.

Results Based on the analysis of results and with respect to the establishment of conditions for equality of variances with Levene's test $(P \geq 0.05)$ and normal distribution of data with Shapiro test $(P \geq 0.05)$, parametric tests were used. Given that research has predicted the relationship, the Pearson correlation test and multiple regressions by Enter method were used by using SPSS23. The results of Pearson correlation showed that domains of emotional validation, superior values, stability, and consensus showed a significant positive correlation with perceived empathy of mothers having children with physical-motor disabilities $(P<0.001)$. It was also found that resiliency has a positive and significant relationship with perceived empathy in mothers with children having physical-motor disabilities $(\mathrm{P}<0.02)$. Multiple regression analysis showed the shared variance emotional schemas and resiliency with perceived empathy is 0.46 . The main findings of regression analysis, via theentermethod, showed that the emotional schema validation, having superior values and resiliency factors have a significant effect in the prediction of perceived empathy of mothers of children with disabilities. Other emotional schemas are not significant impact factors in the prediction of perceived sympathy of mothers with disabled children. Conclusion According to the results, the high resiliency and a positive emotional schemas such as having superior values and validation are predictors of perceived empathy in the mothers of disabled children. This means that the mothers of children with disabilities in dealing with situations when they have more resiliency and and interpret them as positive, are able to communicate more effectively with their surroundings.In this regard, one of the factors is perceived empathy thathas a significantimpactonthedevelopment of personal relationships between individuals and reflects the person's mental health. In addition, it can be used with resiliency and emotional schemas, so therapeutic intervention is implimented with respect to these two variables.

\section{* Corresponding Author:}

Somaye Kazemian, PhD

Address: Department of Counseling, Faculty of Psychology and Educational Sciences, Allameh Tabataba'i University, Tehran, Iran.

Tel: +98 (912) 3935271

E-Mail: somaye.kazemian@yahoo.com 


\title{
ييشبينى همدلى ادراكشده بر اساس طرحوارههاى هيجانى و تابآورى در مادران داراى فرزند

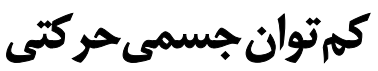

\author{
دانا محمدامينزاده'، "سميه كاظميان'، معصومه اسماعيلى'، يوسف اسمرى'

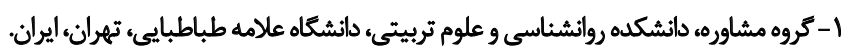

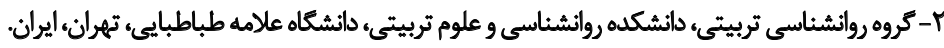

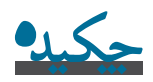

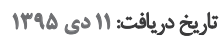

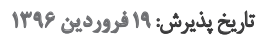

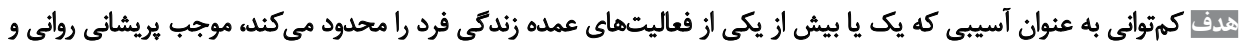

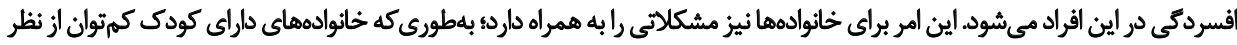

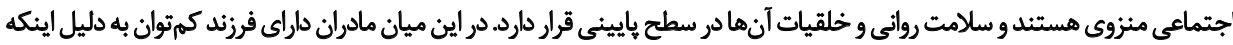

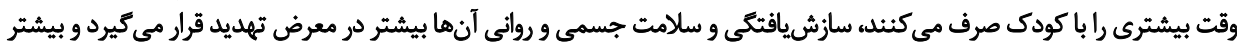

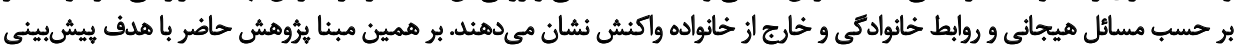

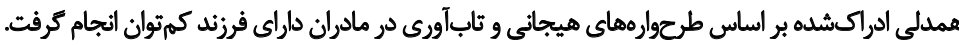

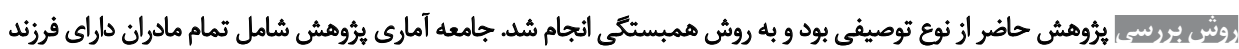
كمتوان جسمى حركتى در سال تحصيلى

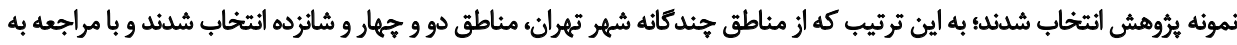

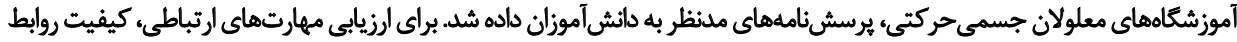

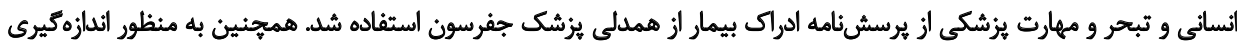

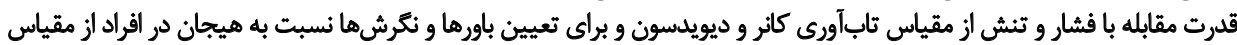

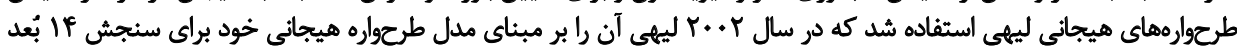
طرحواره تدوين كرده بوده

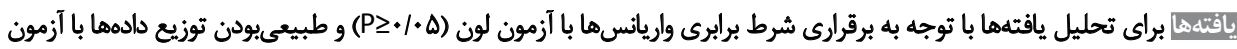

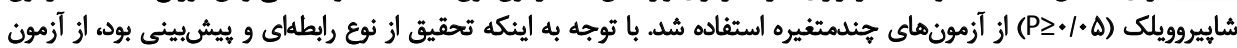

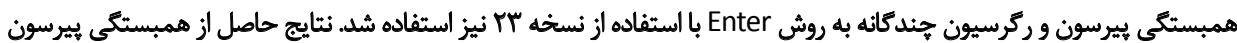

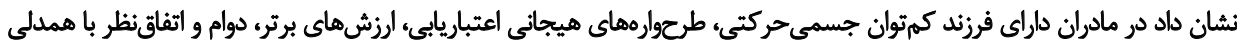

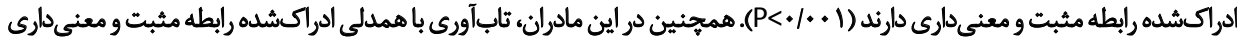

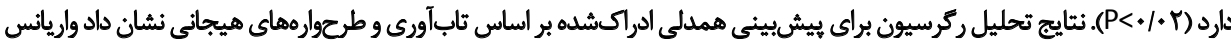

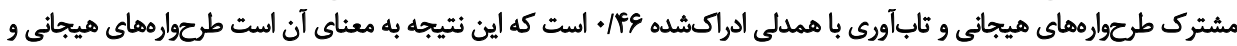

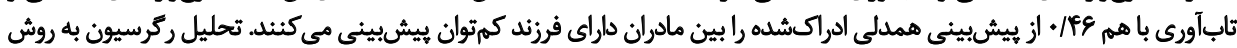

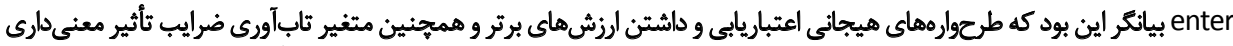

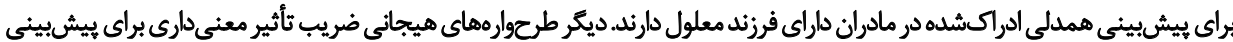
همدلى ادراكشده در مادران فاراي فرزند معلول ندارند.

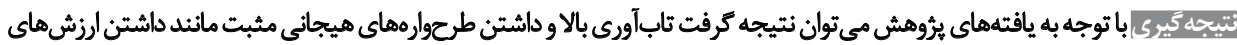

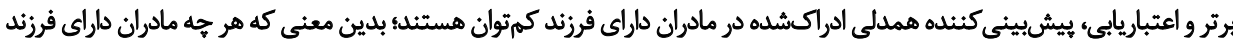

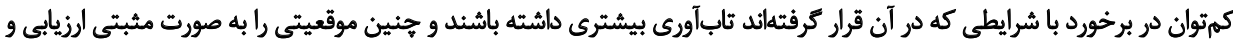

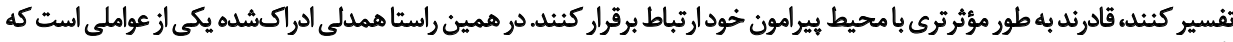

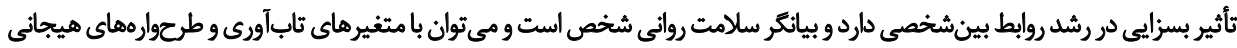

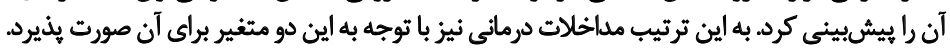

كليدواثهها:

همدلى ادراكشده، طرحوار،هاي هيجاني، تابآورى، مادران دارئ فرزند كمثتوان 


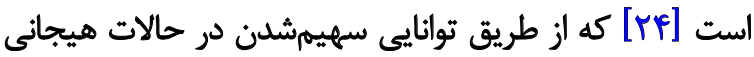

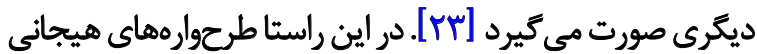

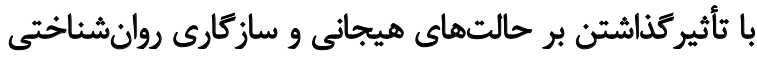

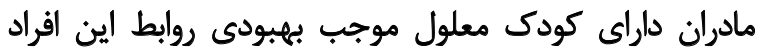

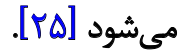

طرحوارههاى هيجانى كه به عنوان طرحها و راهبردهايى

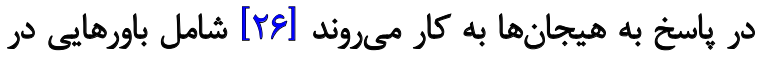

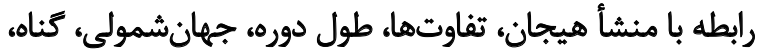

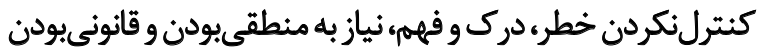

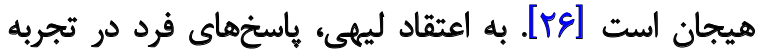

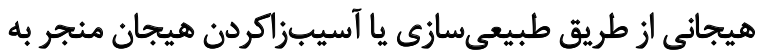

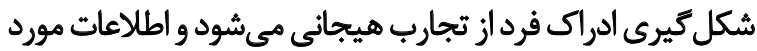

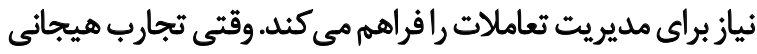

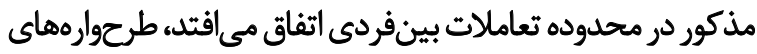

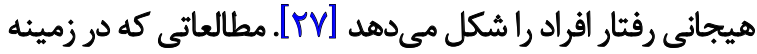

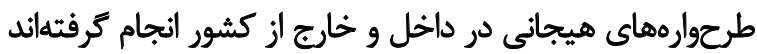

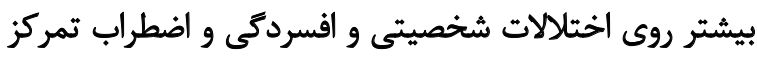

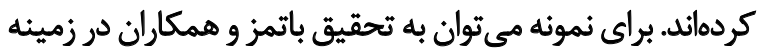

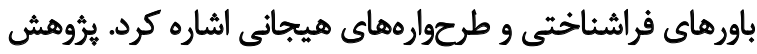

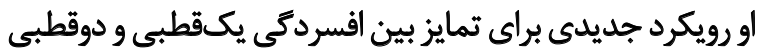

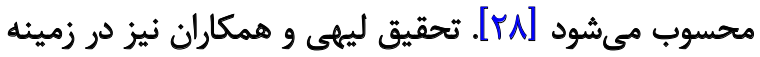

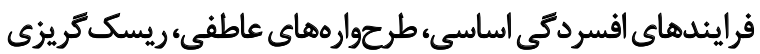

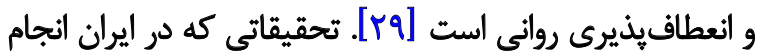

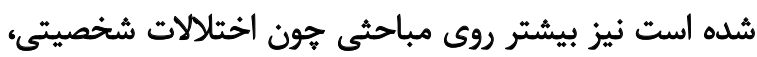

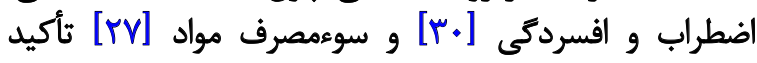

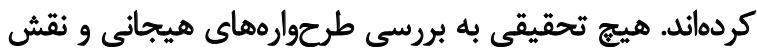

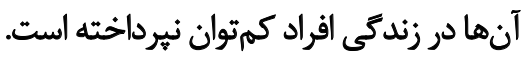

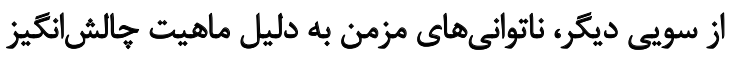

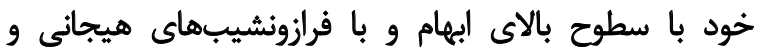

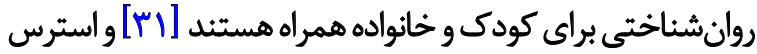

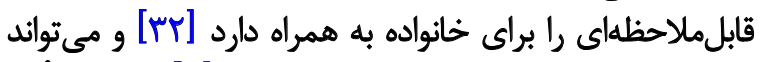

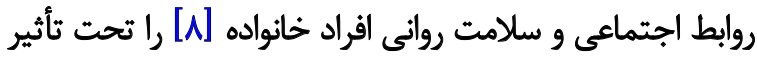

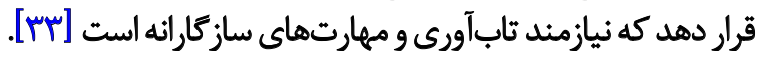

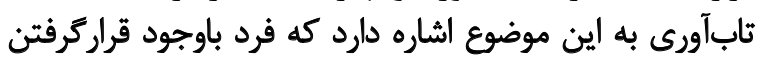

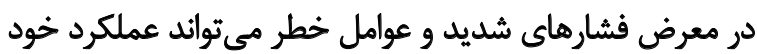

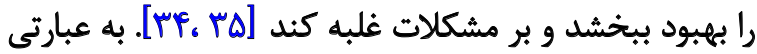

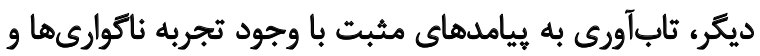

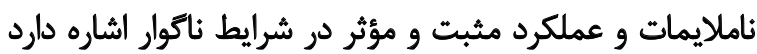

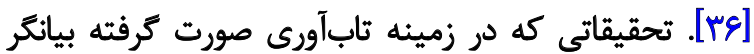

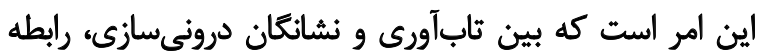

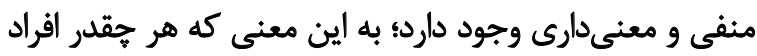

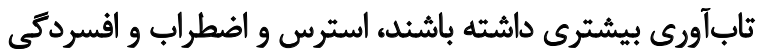

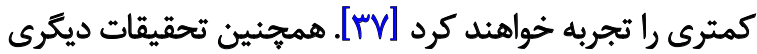

otald

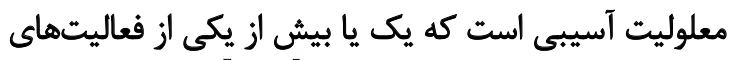

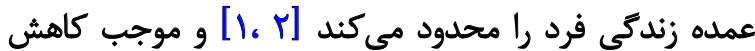

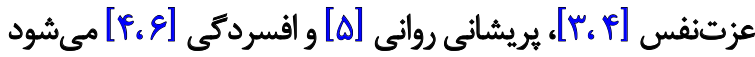

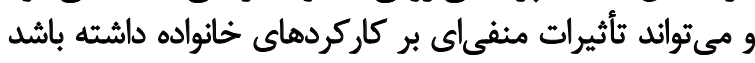

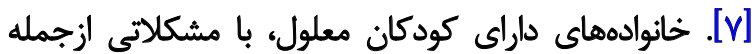

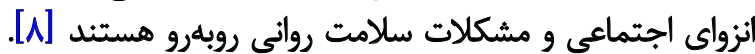

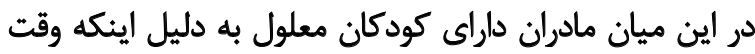

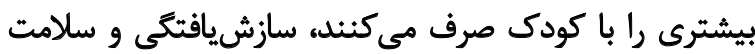

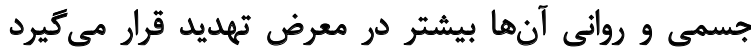

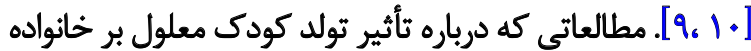

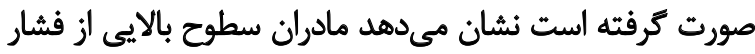

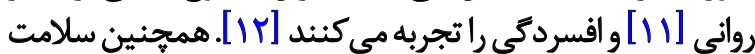

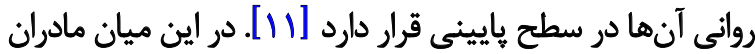

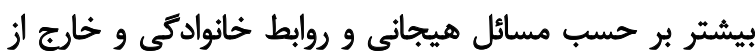

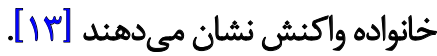

همدلى به عنوان مهارتى بينفردى به معنى توانايى انسان

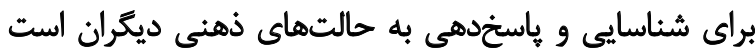

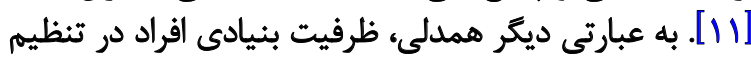

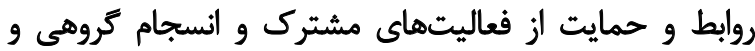

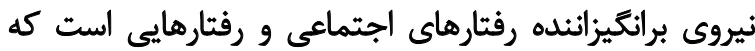

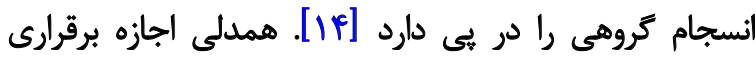

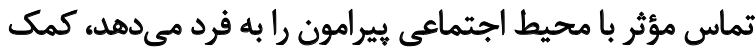

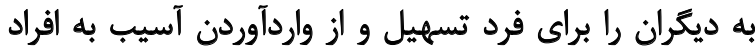

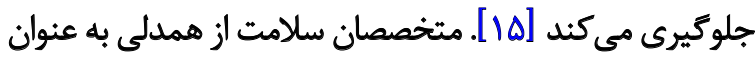

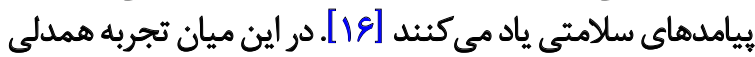

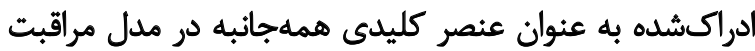

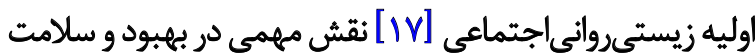

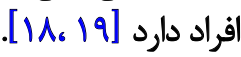

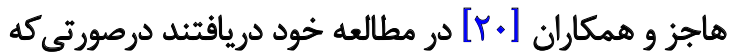

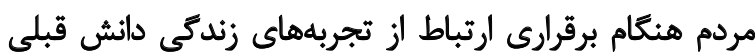

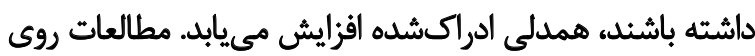

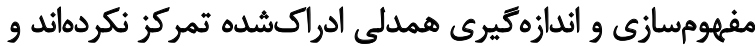

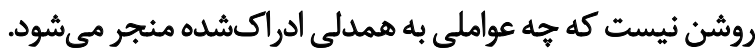

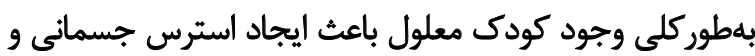

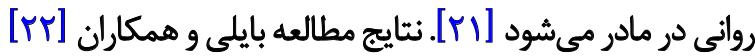

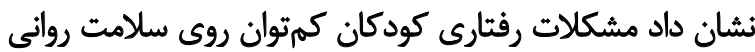

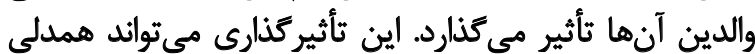

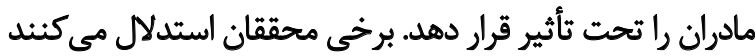

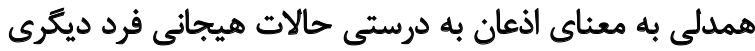

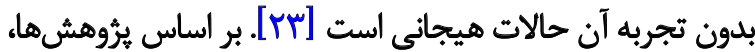

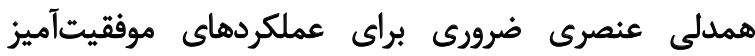
بينشخصى و ياسخدهى هيجانى به احساسات فردى ديكر 
شد يرسشنامه طرحوارههاى هيجانى و تابآورى را به مادران

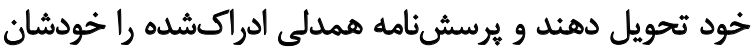

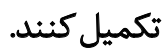

انتخاب اين تعداد از جامعه به عنوان اعضاى نمونه براين مبنا

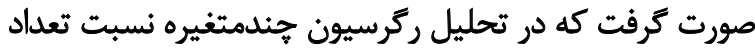

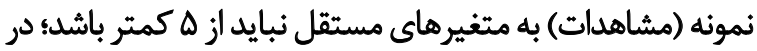

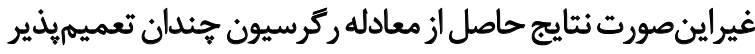

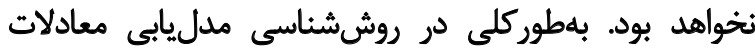

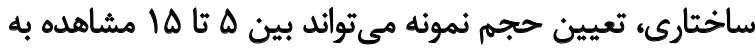

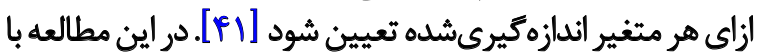

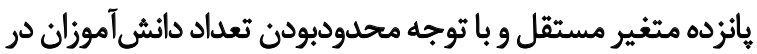

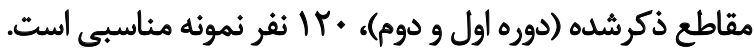

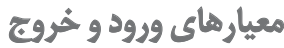

جعيارهاى ورود به مطالعه عبارتند از: دانشآموزان كمتوان

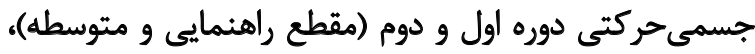

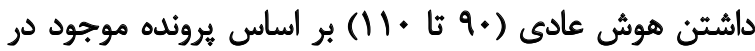

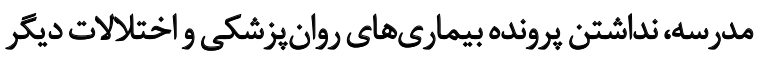

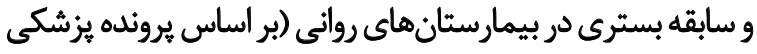

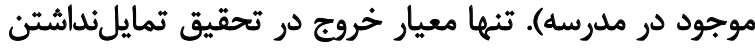

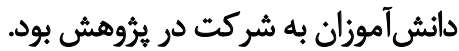

روش

براى انجام اين ثرؤهش، بعد از ترفتن مجوزهاى لازم از

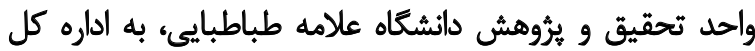

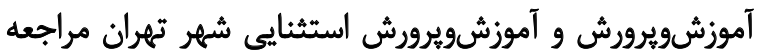

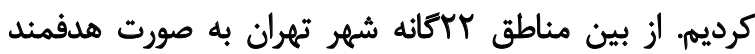

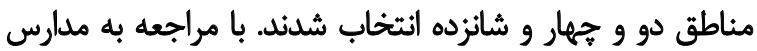

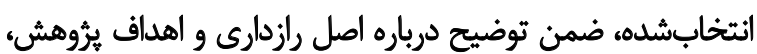

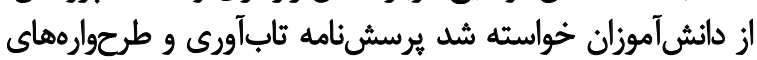

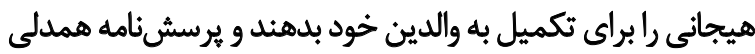

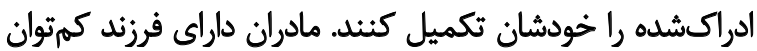

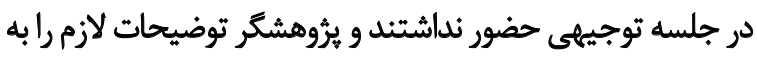

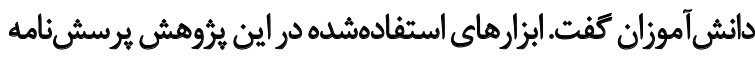
طرحوارههاى هيجانى، تاب آورى و همدلى ادراكشيده بود.

\section{يرسش نامه همدلى ادراكشده}

براى اندازمكيرى همدلى ادراكشده از مقياس ادراك بيمار

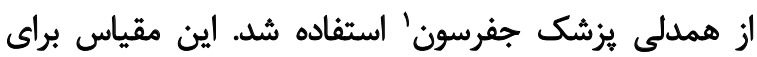
ارزيابى مهارتهاى ارتباطى، كيفيت روابط انسانى و تبحر و

1. Jefferson Scale of Patient's Perceptions of Physician Empathy Questionnaire (JSPPPE)
كه در زمينه تابآورى انجام شده است نشان دادند تابآورى مآدي

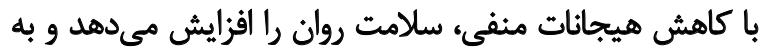

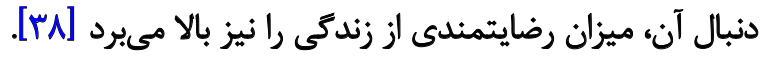

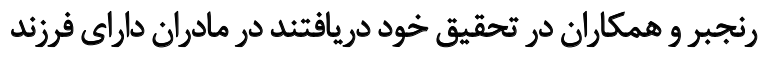

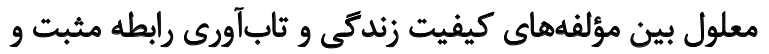

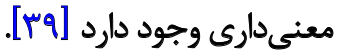

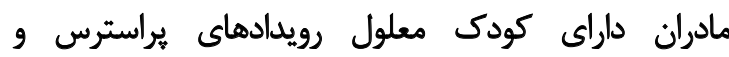

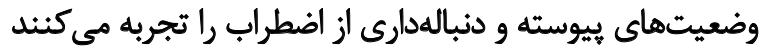

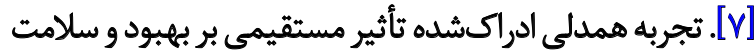

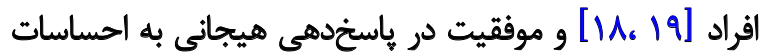

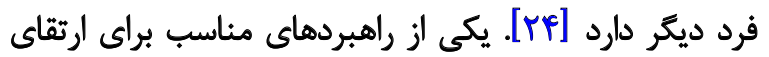

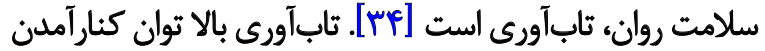

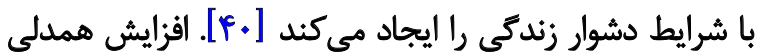

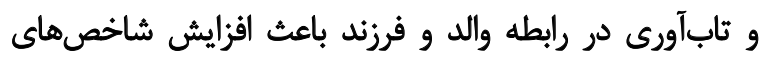

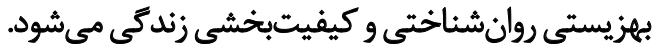
با توجه به مطالب ذكرشده، به منظور بيشبيشينى همدلى

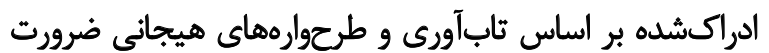

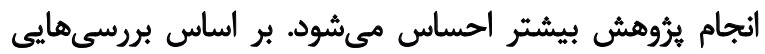

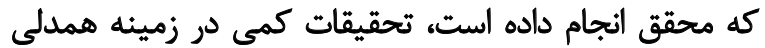

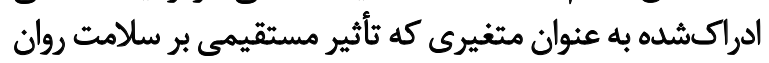

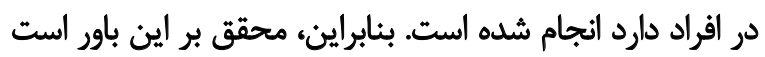

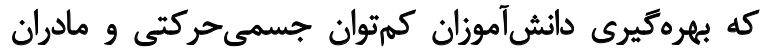

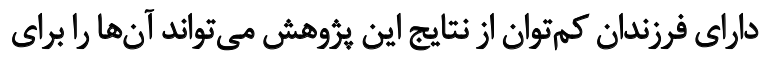

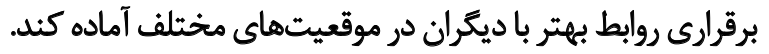

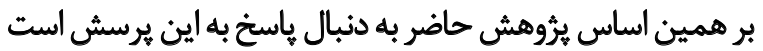

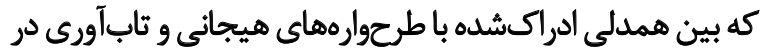

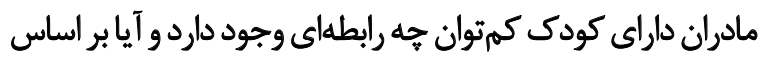

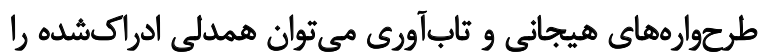

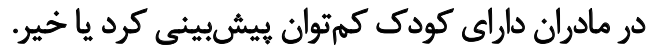

$$
\text { ورش بروسى }
$$

هرؤوهش حاضر از نوع توصيفى و به روش همبستكي است.

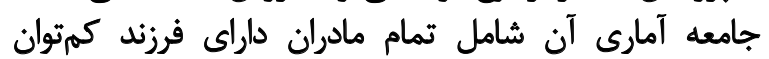

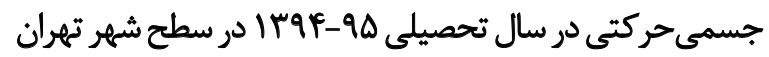

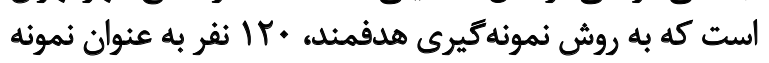

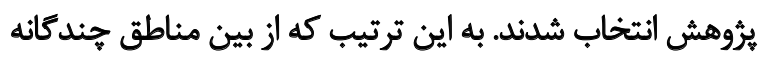

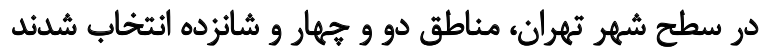

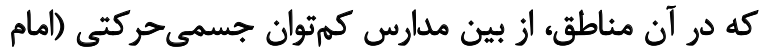

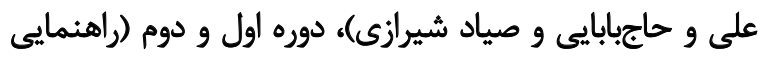

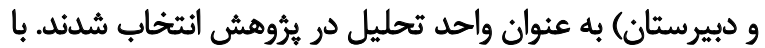

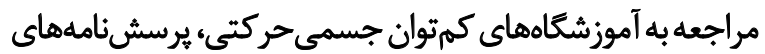

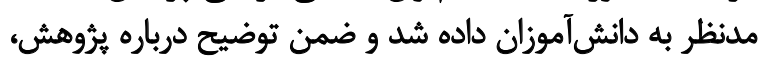

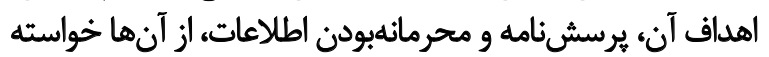




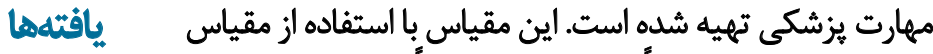
با توجه به برقرارى شرط برابرى واريانسها با آزمون لون إنون

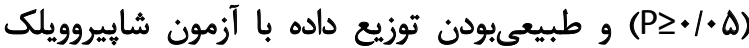

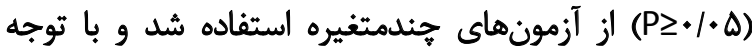

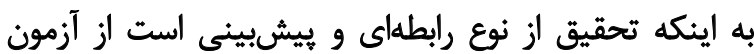

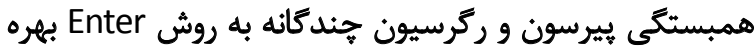

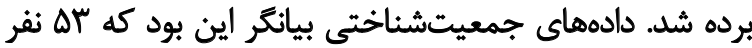

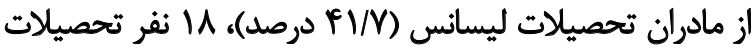

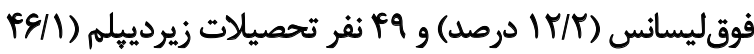

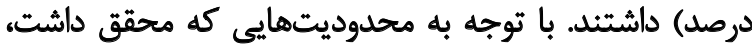

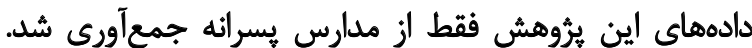

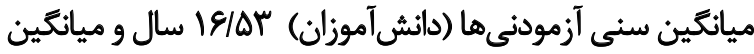

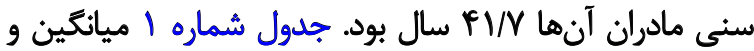
انحراف استاندارد هر يك از متغيرهاي يُروهش ران انشان مي دهاند. جدول شماره ب نتايج آزمون همبستخى طرحوارههاى هيجانى

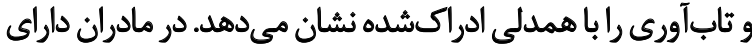

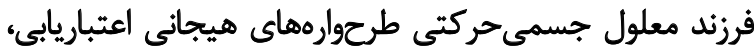

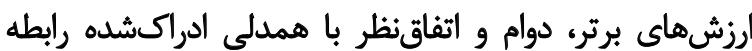

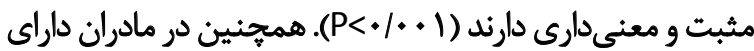

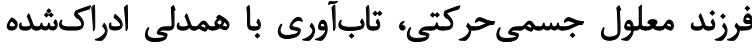

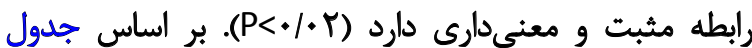

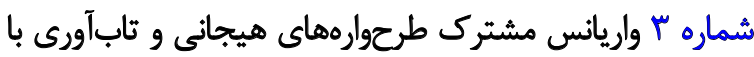
همدلى ادراكشده

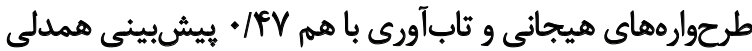

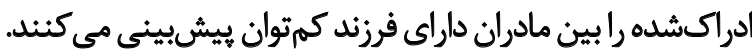

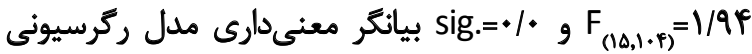
محاسبهشده از لحاظ آمارى در تحقيق حاضر است.

جدول شماره \& نتايج تحليل ضرايب ركرسيونى را نشان

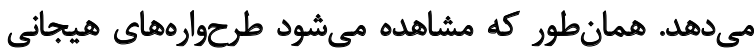

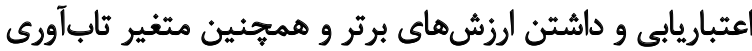

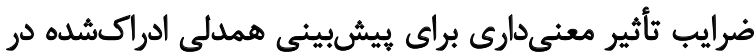

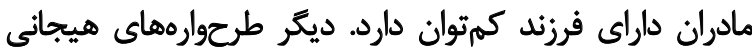

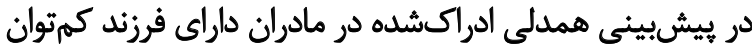
ضريب تأثير معنى دارى ندارند.

بحث

هدف يرؤهش حاضر بيشبينى همدلى ادراكشده بر اساس

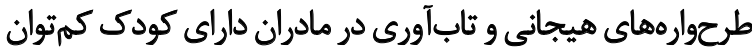

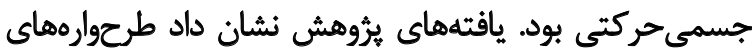

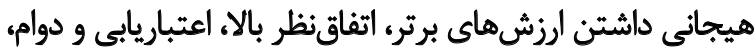

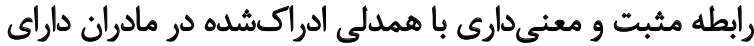

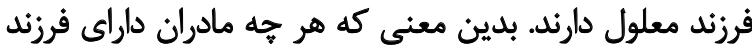

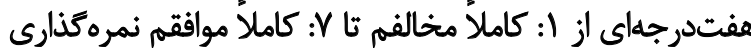

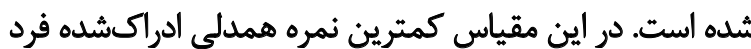

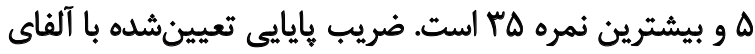

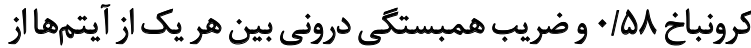

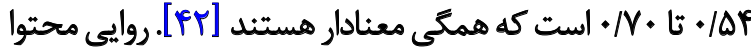

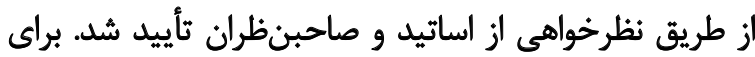

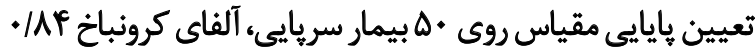

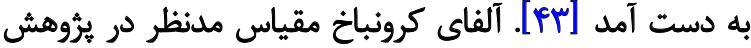

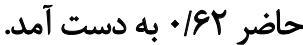

\section{يرسش نامه طرحوارههاى هيجانى}

براي اندازمكيرى طرحوارههاى هيجانى از مقياس طرحوارههاى

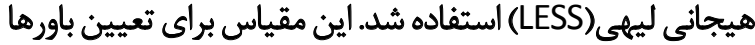

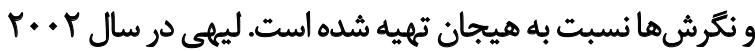

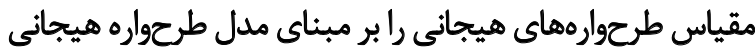

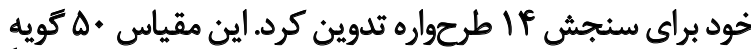

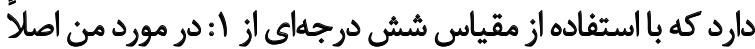

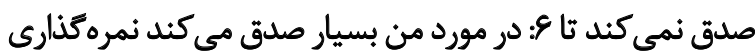

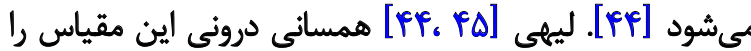

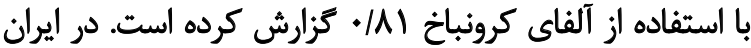

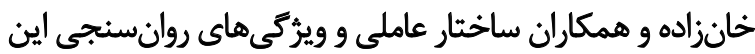

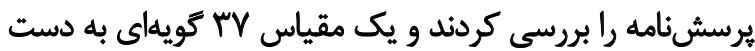

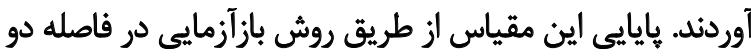

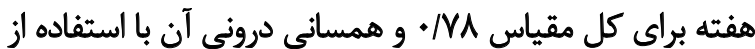

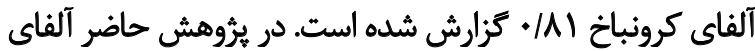
كرونباخ يرسشنامه مذكور /N/ • به دست آمد.

يرسش نامه تاب آورى

براى اندازمخيرى تابآورى از مقياس تابآورى كانر ديويدسون

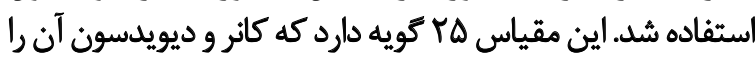

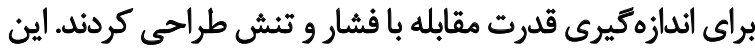

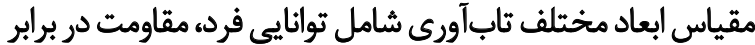

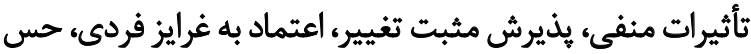

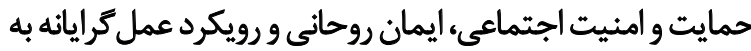

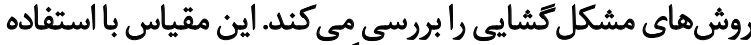

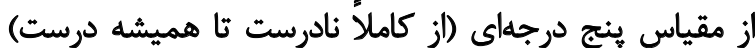

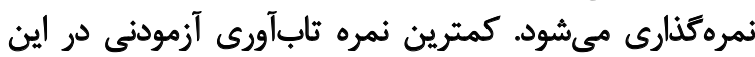

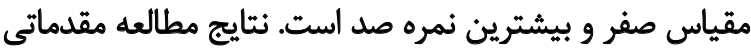

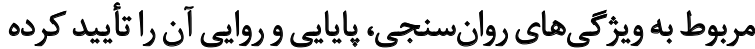

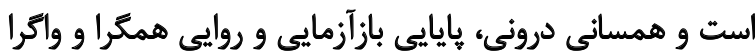

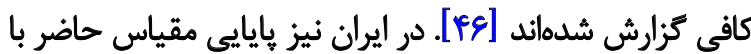

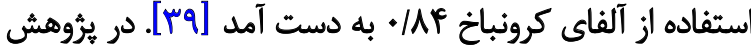

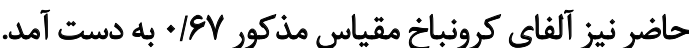


ديكَر، هيجانات مثبت منابع روانشناحتى بسيار مهمي هستند

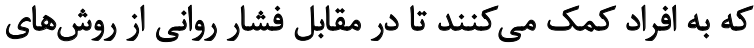

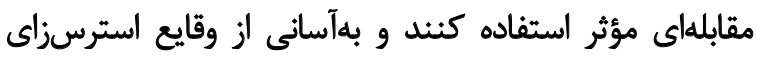

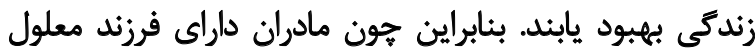

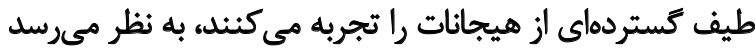

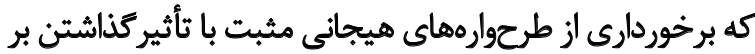

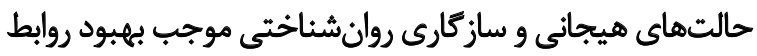

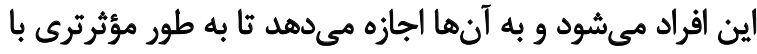

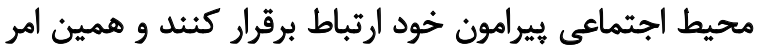
كمك كردن به ديكران را براى آنها آسانتر مى كنداط.

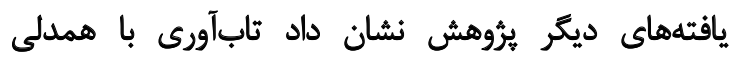

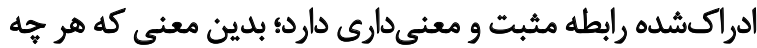

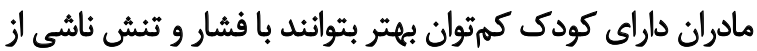

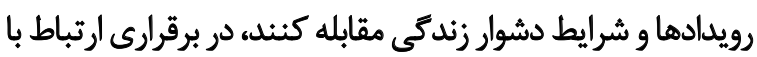

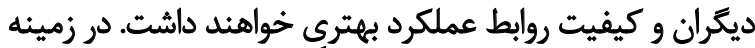

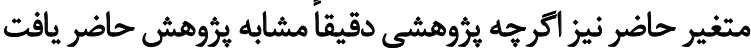

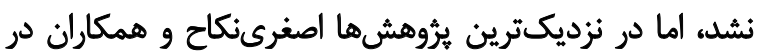

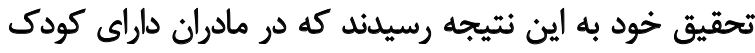

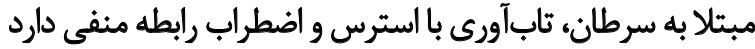

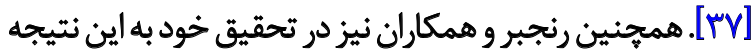

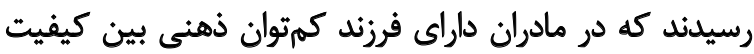

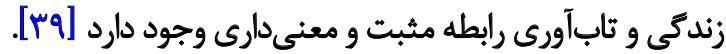

معلول در طرحوارههاى هيجانى داشتن ارزش هاى برترء اتفاقنظر

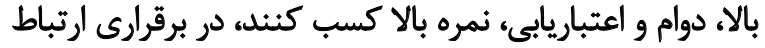

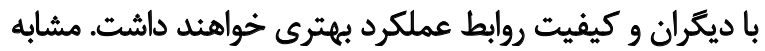

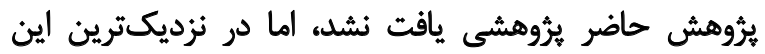

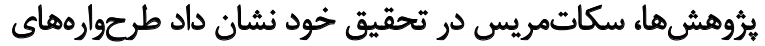

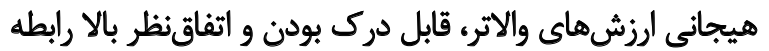

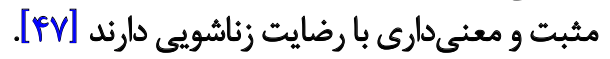

ليهي در تحقيق خود در زمينه رضايتمندى در روابط صميمى

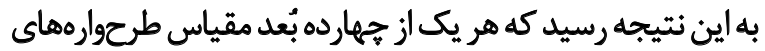

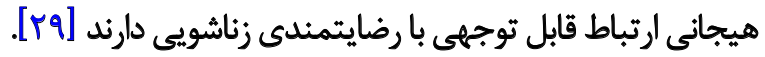

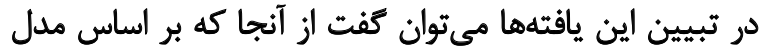

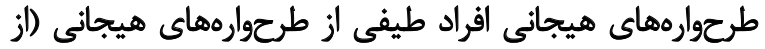

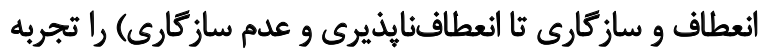

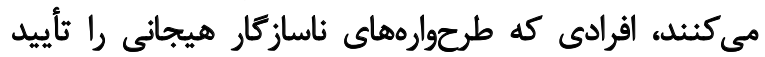

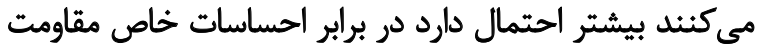

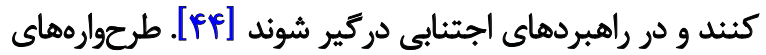

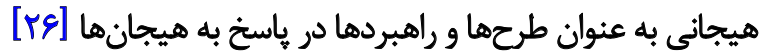

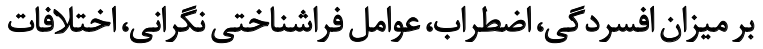
زناشويى، كاهش تمركز حواس و اختلال شخصيت تأثير دارند.

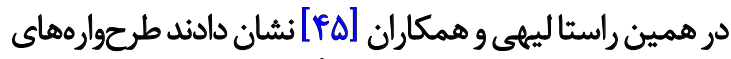

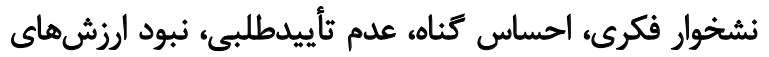

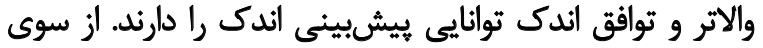

جدول ا. ميانكين و انحراف استاندارد متغيرهاى تحقيق

\begin{tabular}{|c|c|c|}
\hline اتحراف استاندارد & مياتكين & متغير \\
\hline $1 / 199$ & $\pi / / R$ & هملى \\
\hline VIr & $A T / M$ & تابأورى \\
\hline$M / 1$ & IVar & اعتباريايى \\
\hline T/GT & IV/FD & قابل فهم \\
\hline T/FE & $\mathbb{N} / \mathrm{N}$ & احساس كناه \\
\hline $1 / \mathrm{Na}$ & $|g / \pi|$ & سادانكارى \\
\hline$V / \Delta \Delta$ & $1 . / \% 1$ & ارزش برتر \\
\hline $1 / 91$ & $1 . / 19$ & كنترل \\
\hline$M$ & V/AY & كرختى \\
\hline $1 / 8$. & $1 \% / \%$. & خردمرايى \\
\hline $1 / \pi$ & v/ro & دوام \\
\hline$r / N$ & $1 w / 9 r$ & اثفاتنظر \\
\hline$r / 9)$ & $r Y / \bullet A$ & بذيرش \\
\hline$r / T r$ & $19 / A$. & نشخوار فكرى \\
\hline $1 / 4$. & $V / N A$ & ابراز \\
\hline$I M T$ & NTE & سرزنش \\
\hline
\end{tabular}


جدول r. ماتريس همبستكى طحوارههاي هيجاني و ثاب آورى با همدلى ادراكشده

\begin{tabular}{|c|c|c|c|c|c|c|c|c|c|c|c|c|c|c|c|c|}
\hline 17 & 10 & $1 E$ & ir & Ir & 11 & 1. & 9 & $\wedge$ & $\checkmark$ & 7 & 0 & $\varepsilon$ & $r$ & $r$ & 1 & 管; \\
\hline & & & & & & & & & & & & & & & 1 & 5 \\
\hline & & & & & & & & & & & & & & 1 &.$M I^{*}$ & $\frac{\sqrt{6}}{\bar{c}}$ \\
\hline & & & & & & & & & & & & & 1 & $.19 r^{* *}$ &.$/ 4 \Delta^{* * *}$ & 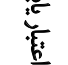 \\
\hline & & & & & & & & & & & & 1 &.$/ 19^{*}$ & .1 .8 & 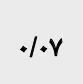 & 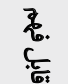 \\
\hline & & & & & & & & & & & 1 &.$- / 1 f$ &.$/ N$ & . &.$/ r$ & \\
\hline & & & & & & & & & & 1 &.$/ 11$ & $-* 1 * 1$ & . &.$/ T r^{* *}$ & $-.1+1$ & $\frac{\pi}{6}$ \\
\hline & & & & & & & & & 1 & $+/ 19^{\circ}$ & $* / T$ & . & $+/ M^{*}$ & $+/ \Delta 0^{\circ * *}$ & 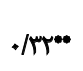 & $\begin{array}{l}x_{z} \\
x_{z} \\
c_{z}\end{array}$ \\
\hline & & & & & & & & 1 &.$- / \mu$ & $\cdot / \mathrm{A}^{*}$ &.$/ T \lambda^{* *}$ & $.1+\Delta$ & . $1 . \mu$ & $\bullet / \mu_{*} * *$ & $-.1 . \Delta$ & 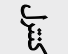 \\
\hline & & & & & & & 1 & 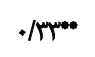 & $\cdot M^{*}$ & $.1 \cdot 1$ & $-.1 . r$ & $M$ & 11 & 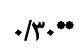 & $-.1 . r$ & 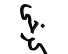 \\
\hline & & & & & & 1 & 11 & $-\cdot / r_{*}^{*}$ & -.1 .8 & $\cdot / 1$ & . rene* &.$- / 10$ & -.1 .8 & $.1 \mathrm{~A}^{*}$ &.$/ 4$ & $\frac{2}{5}$ \\
\hline & & & & & 1 & $-\cdot / M$ &.$- / . \gamma$ &.$/ / \gamma^{a *}$ & $. / 0)^{p e n}$ & $.1+0$ &.$- / \mathrm{N}^{* *}$ & 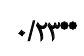 & o/pee & $\% A$ &.$/ *_{*}^{*}$ & $\frac{t}{t}$ \\
\hline & & & & 1 & . & .1 .9 & $. / \% T=$ & $\cdot / N r$ & $\cdot N \Lambda^{* *}$ &.$/ 1 F$ &.$/ r$ &.$/ \pi r^{* *}$ & $+/ \Delta+*$ &.$/ 40^{\circ *}$ &.$M \mathrm{~A}^{*}$ & 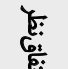 \\
\hline & & & 1 & $.1 . V$ &.$M 11$ &.$/ \Gamma \omega^{* *}$ &.$- / \cdot \Delta$ & $\% \gamma$ &.$/ R$ & .1 & $\cdot / \Delta^{* *}$ & $-\% / \gamma$ &.$/ 10$ &.$/ T^{* * *}$ &.$/ 1 F$ & $\begin{array}{l}c_{z} \\
z \\
z\end{array}$ \\
\hline & & 1 & $\cdot / 4 \lambda^{*}$ & . &.$/ 18$ & .1 &.$/ \Delta_{0} \cdots$ &.$/ Y$ &.$/ I V$ & -.1 .1 & . & - Epeow & .1 .8 & .1 .9 & .1 .01 & $\frac{\hbar}{6}$ \\
\hline & 1 &.$/ F q^{* *}$ & $\theta /+r$ & $+/ \pi \lambda^{* *}$ & $+/$ Trea* & $-t / 1 T$ & 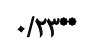 & $. \mid 8)^{* *}$ & $+1 . \Delta$ &.$/ F F$ & $-+119^{*}$ & $+/ \pi+*$ & $* / *$ &.$/ I^{\circ}$ & $-+1+4$ & $\underline{\underline{k}}$ \\
\hline 1 &.$/ N r$ & -.10 &.$/ 1 \Delta$ & 10 & $\cdot M I^{*}$ &.$M I^{\bullet}$ &.$- / M$ & 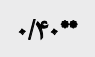 &.$/ \mathrm{A}^{*}$ &.$/ \pi r^{*}$ & - & $-\infty 1.0$ &.$/ 40^{*}$ & 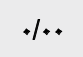 &.$/ 18$ & 魷 \\
\hline
\end{tabular}

توانبخننى

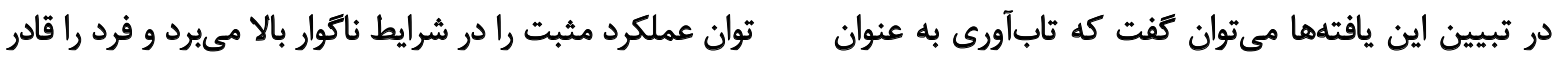

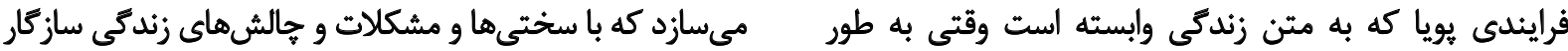

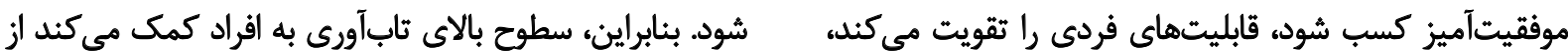


جدول r . خلاصه مدل ركرسيونى

\begin{tabular}{|c|c|c|c|c|c|c|}
\hline Sig. & $\mathbf{f}$ & خطاى معيار & مجذور R تصحيحشده & $\mathbf{R}^{2}$ & $\mathbf{R}$ & 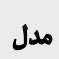 \\
\hline $.1 . r$ & 1/9F & Whif & $J+\gamma$ &.$/ M 1 q$ &.$/ F \& A$ & \\
\hline
\end{tabular}

توانبخننى

جدول ج. نثايج تحليل ضرايب ركرسيون

\begin{tabular}{|c|c|c|c|c|c|}
\hline \multirow{2}{*}{ Sig. } & \multirow{2}{*}{$\mathbf{t}$} & \multirow{2}{*}{ ضرايب استاندارد } & \multicolumn{2}{|c|}{ ضرايب غير استاندارد } & \multirow{2}{*}{ مدل مدل } \\
\hline & & & خطاى انحراف معيار & B & \\
\hline $.1 . .1$ & VITAT & & $r / F E A$ & YA/TAS & ثابت \\
\hline $.1 . r$ & r/aro &.$/ 941$ & $\mid \mp 91$ & V/MID & تاببآورى \\
\hline .1 .4 & r/NPq & ./VEQ & $\cdot / 4 \lambda$ & $V \cdot r q$ & اعتباريابى \\
\hline.$/ 49$ & $V .8 T$ &.$/ T M$ &.$/ 10$ & .1109 & قابل فهم بودن \\
\hline.$/ 19$ &.$- / 11 V$ & $-.|| A \mid$ & . Mer & $-* / 1 \cdot r$ & احساس كثاه \\
\hline .1 .9 & $-1 / F W$ &.$/ 194$ & .1 .28 & $-+\mid 191$ & سادمانكارى \\
\hline$\%$ & $\% / \cdot 10$ &.$/ \pi$ &.$/ 9 \Delta S$ & INAT & الرزشهاى برتر \\
\hline.$/ 4 \pi$ &.$- /$ MAP &.$- / \operatorname{ket}$ &.$|+8|$ &.$- \mid$ | & كتترل \\
\hline$\cdot M r$ &.$/ N \Delta$ &.$/ 1 \Delta E$ &.$/ e q \gamma$ &.$/ 1 n t$ & كرخى \\
\hline$\cdot M r$ & $-+/ T \Delta T^{\circ}$ & $-.1 \cdot \Delta V$ &.$/ 1 P q$ & $-* 1 \cdot \Delta r$ & خرديرابودن \\
\hline - ART & $-* / M T$ & $-* / 1 r$ & +/ANF & $-+/ 179$ & 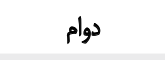 \\
\hline .119 & $1 / F+r$ & $\cdot|+N|$ & $. / M+1$ & גזות & اتفاقنظر \\
\hline . IFA &.$/ 4 \cdot r$ & $.8 \%+$ &.$/ 194$ &. & يذيرش احساسات \\
\hline.$/ \pi T$ & $-* \mid 41$ & -.1870 & $. / F \mid$ & $-+/ 4+1$ & نشخوار فكرى \\
\hline.$r A$ & - IAYA &.$/ \pi$ &.$/ M M$ &.$M P$ & ابراز احساسات \\
\hline.$/ 1 F$ & V/rVA & TrT/ &.$/ 197$ & . /rAY & سرزئش \\
\hline
\end{tabular}

به احساسات ناشى از تفسير فرد از يك رويداد به كار مىروند

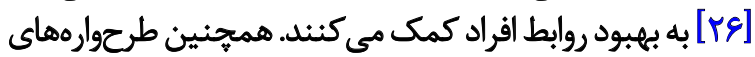

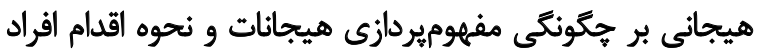

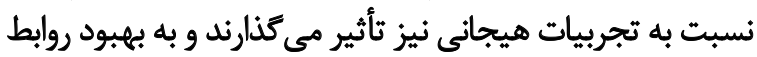

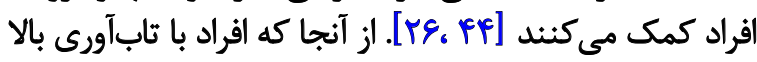

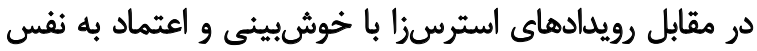

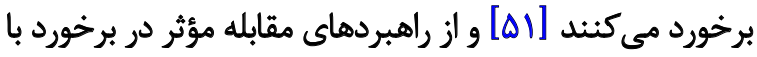

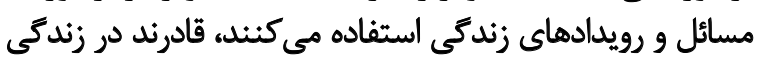
اجتماعى جوابكوى حالتهاى هيجانى ديكران باشئد و هيجانات

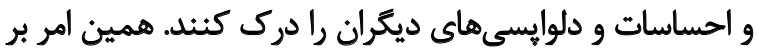

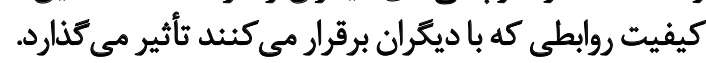

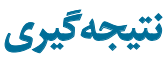

با توجه به يافتههاى ثرُوهش ميثتوان نتيجه كرفت ثابآورى

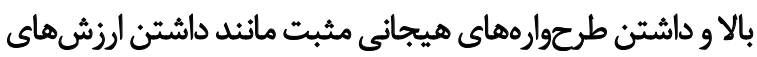

عواطف و هيجانهاى مثبت به منظور هشتسرنهادن تجربه بهاى

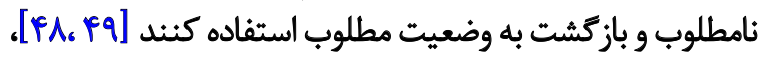

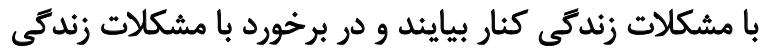

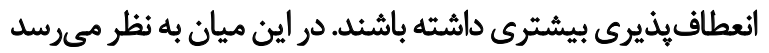

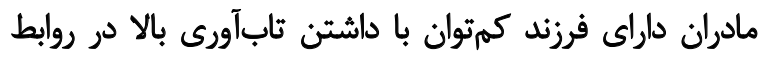

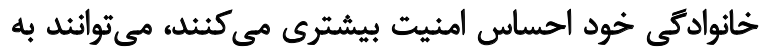

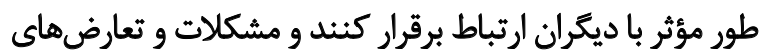

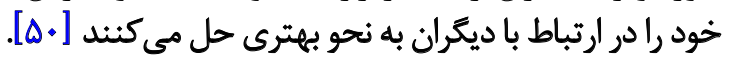
همجنين نتايج تحليل ركرسيون براى بيشبيني همدلي

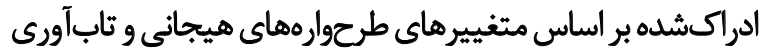

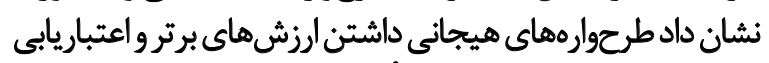

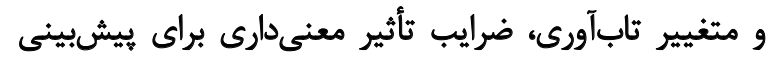

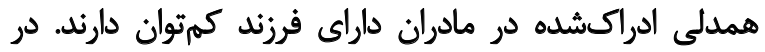

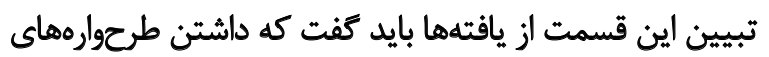

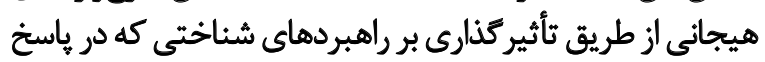


برتر و اعتباريابى ييشبينى كنينده هملالى ادراكشده در مادران

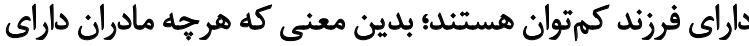

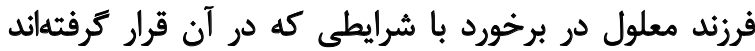

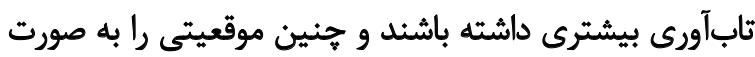

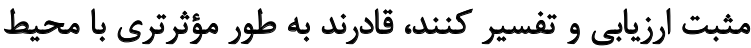
بيرامون خود ارتباط برقرار كنئد.

در خايان يُشنهاد مىشود نقش همدلى ادراكشده با ديكر

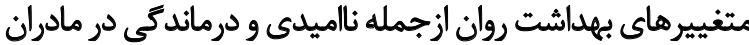

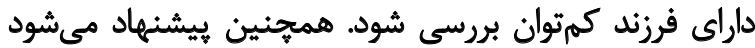

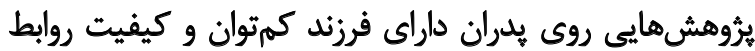

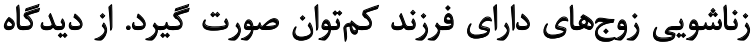

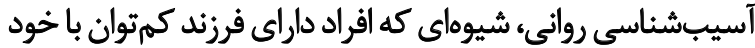

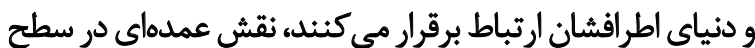

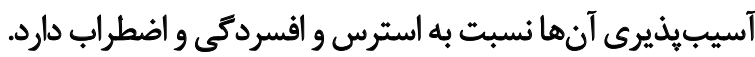

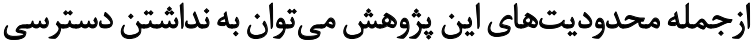
مستقيم به مادران داراى فرزند كمثتوان اشاره كرد.

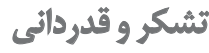

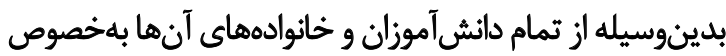

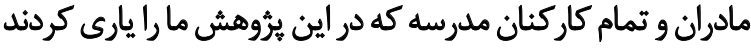

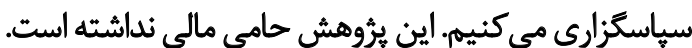




\section{References}

[1] Brown RL, Turner RJ. Physical limitation and anger. Society and MentalHealth.2012;2(2):69-84. doi:10.1177/2156869312444441

[2] Fathi K, Adibsereshki N, Sajedi F. [The effectiveness of emotional intelligence training on aggression and hyperactivity of students with physical disability (Persian)]. Archives of Rehabilitation. 2014; 14(6):129-35

[3] Nosek MA, Hughes RB, Swedlund N, Taylor HB, Swank P. Selfesteem and women with disabilities. Social Science \& Medicine. 2003; 56(8):1737-47. doi: 10.1016/s0277-9536(02)00169-7

[4] Salehi M, Kharaz Tavakol H, Shabani M, Ziaei T. The relationship between self-esteem and sexual self-concept in people with physical-motor disabilities. Iranian Red Crescent Medical Journal. 2015; 17(1):25359. doi: 10.5812/ircmj.25359

[5] Dadkhah A. [The effect of psychorehabilitation program on children with physical and mental disability (Persian)]. Archives of Rehabilitation. 2007; 8(2):57-62

[6] Sharifian-Sani M, Sajjadi H, Tolouei F, Kazem-Nezhad A. [Girls and women with physical disabilities: Needs and problems (Persian)]. Archives of Rehabilitation. 2006; 7(2):41-8.

[7] Gau SS-F, Chou MC, Chiang HL, Lee JC, Wong CC, Chou WJ, et al. Parental adjustment, marital relationship, and family function in families of children with autism. Research in Autism Spectrum Disorders. 2012; 6(1):263-70. doi: 10.1016/j.rasd.2011.05.007

[8] Khodabakhshi Koolaee A, Derakhshandeh M. [Effectiveness of hope-oriented group therapy on life meaning and resilience in mothers with physical-motor disabled children (Persian)]. Iranian Journal of Pediatric Nursing. 2015; 1(3):15-25.

[9] Gupta A, Singhal N. Positive perceptions in parents of children with disabilities. Asia Pacific Disability Rehabilitation Journal. 2004; 15(1):22-35.

[10] Ashori M, Afrooz GA, Arjmandnia AA, Pourmohamadreza-Tajrishi M, Ghobari-Bonab B. [Group positive parenting program (Triple-P) and the relationships of mother-child with intellectual disability (Persian)]. Practice in Clinical Psychology. 2015; 3(1):31 8.

[11] Zembat R, Yildız D. A comparison of acceptance and hopelessness levels of disabled preschool children's mothers. Procedia-Social and Behavioral Sciences. 2010; 2(2):1457-61. doi 10.1016/j.sbspro.2010.03.218

[12] Emerson E, Llewellyn G. The mental health of Australian moth ers and fathers of young children at risk of disability. Australian and New Zealand Journal of Public Health. 2008; 32(1):53-9. doi: 10.1111/j.1753-6405.2008.00166.x

[13] Kouhsali M, Mirzamani Bafghi M, Mohamadkhani P, Karimloo M. [Comparison of social adjustment between mothers of educable mentally retarded girls and mothers of normal girls in Kashan (Persian)]. Archives of Rehabilation. 2007; 8(29):40-5.

[14] Rieffe C, Ketelaar L, Wiefferink CH. Assessing empathy in young children: Construction and validation of an empathy questionnaire (EmQue). Personality and Individual Differences. 2010; 49(5):362-7. doi: 10.1016/j.paid.2010.03.046
[15] Baron-Cohen S, Wheelwright S. The empathy quotient: An investigation of adults with asperger syndrome or high functioning autism, and normal sex differences. Journal of Autism and Developmental Disorders. 2004; 34(2):163-75. doi: 10.1023/b:ja dd. 0000022607.19833 .00

[16] Hurter S, Paloyelis Y, Williams AC, Fotopoulou A. Partners' empathy increases pain ratings: Effects of perceived empathy and attachment style on pain report and display. Journal of Pain. 2014; 15(9):934-44. doi: 10.1016/j.jpain.2014.06.004

[17] Nambisan P. Information seeking and social support in online health communities: Impact on patients' perceived empathy. Journal of the American Medical Informatics Association. 2011; 18(3):298-304. doi: 10.1136/amiajnl-2010-000058

[18] Price S, Mercer SW, MacPherson H. Practitioner empathy, patient enablement and health outcomes: A prospective study of acupuncture patients. Patient Education and Counseling. 2006; 63(1-2):239-45. doi: 10.1016/j.pec.2005.11.006

[19] Rakel DP, Hoeft TJ, Barrett BP, Chewning BA, Craig BM, Niu M. Practitioner empathy and the duration of the common cold. Family Medicine. 2009; 41(7):494. PMCID: PMC2720820

[20] Hodges SD, Kiel KJ, Kramer ADI, Veach D, Villanueva BR. Giving birth to empathy: The effects of similar experience on empathic accuracy, empathic concern, and perceived empathy. Personality and Social Psychology Bulletin. 2010; 36(3):398-409. doi: 10.1177/0146167209350326

[21] Schieve LA, Blumberg SJ, Rice C, Visser SN, Boyle C. The relationship between autism and parenting stress. Pediatrics. 2007; 119(1):114-21. doi: 10.1542/peds.2006-2089q

[22] Bailey DB, Golden RN, Roberts J, Ford A. Maternal depression and developmental disability: Research critique. Mental Retardation and Developmental Disabilities Research Reviews. 2007; 13(4):321-9. doi: 10.1002/mrdd.20172

[23] Birhanu Z, Assefa T, Woldie M, Morankar S. Predictors of perceived empathy among patients visiting primary health-care centers in central Ethiopia. International Journal for Quality in Health Care. 2012; 24(2):161-8. doi: 10.1093/intqhe/mzs001

[24] Barzegar K. [Influence of teaching empathy on aggression and social adjustment of girl students (Persian)]. Research in School and Virtual Learning. 2015; 3(9):21-32.

[25] Zandvanian Naeini A, Morowati Sharifabad MA, Amrollahi M. [Efficacy of anger management training on increasing mental health of disabled children mothers (Persian)]. Toloo-e-Behdasht. 2014; 13(3):73-85.

[26] Leahy RL. Emotional schemas and resistance to change in anxiety disorders. Cognitive and Behavioral Practice. 2007; 14(1):3645. doi: 10.1016/j.cbpra.2006.08.001

[27] Hasani J, Tajodini E, Ghaedniyaie Jahromi A, Farmani Shahreza $\mathrm{S}$. [The assessments of cognitive emotion regulation strategies and emotional schemas in spouses of people with substance abuse and spouses of normal people (Persian)]. Journal of Clinical Psycology. 2014; 6(1):91-101.

[28] Batmaz S, Ulusoy Kaymak S, Kocbiyik S, Turkcapar MH. Metacognitions and emotional schemas: A new cognitive perspective for the distinction between unipolar and bipolar depression. 
Comprehensive Psychiatry. 2014; 55(7):1546-55. doi: 10.1016/j. comppsych.2014.05.016

[29] Leahy RL. Introduction: Emotional schemas, emotion regulation, and psychopathology. International Journal of Cognitive Therapy. 2012; 5(4):359-61. doi: 10.1521/ijct.2012.5.4.359

[30] Rahabarian M, Mohammadi A, Abasi I, Meysamie A, Nejatisafa A. A comparison of emotional schemas in patients with bipolar disorders and major depressive disorder in remission and nonclinical population. Practice in Clinical Psychology. 2016; 4(2):121-128. doi: 10.15412/j.jpcp.06040207

[31] Fletcher D, Sarkar M. Psychological resilience. European Psychologist. 2013; 18(1):12-23. doi: 10.1027/1016-9040/a000124

[32] Ergün S, Ertem G. Difficulties of mothers living with mentally disabled children. Development. 2012; 1(3):213-30.

[33] Hosini Ghomi T, Ebrahimi Ghavam S, Oloumi Z. [Effectiveness of resilience training on level of quality of life of mothers whose children, were between 10-13, suffer from cancer in Imam Khomeini hospital of Tehran during the years 1388-1389 (Persian). Journal of Clinical Psychology. 2011; 1(1):77-100.

[34] Hu T, Zhang D, Wang J. A meta-analysis of the trait resilience and mental health. Personality and Individual Differences. 2015; 76:18-27. doi: 10.1016/j.paid.2014.11.039

[35] Nikrah N, Zare M, Khakbaz H. [Effectiveness of social problem-solving group training on increasing the resiliency of mothers with addicted children (Persian)]. Social Welfare. 2015; 15(56):185-199.

[36] Schoon I. Risk and resilience: Adaptations in changing times. Cambridge: Cambridge University Press; 2006.

[37] Asghari Nekah SM, Jansooz F, Kamali F, Taherinia S. [The resiliency status and emotional distress in mothers of children with cancer (Persian)]. Journal of Clinical Psychology. 2015; 7(1):15-26.

[38] Aslani L, Azkhosh M, Movallali G, Younesi SJ, Salehy Z. [The effectiveness of resiliency training program on the components of quality of life in mothers with hearing-impaired children (Persian)]. Journal of Research \& Method in Education (IOSRJRME). 2014; 4(3):62-6. doi: 10.9790/7388-04336266

[39] Hagh Ranjbar F, Kakavand AR, Borjali A, Bermas H. [Resilience and life quality in mothers of children with mental retardation (Persian)]. Journal of Health Psychology. 2011; 1(1):177-87.

[40] Sood S, Bakhshi A, Devi P. An assessment of perceived stress, resilience and mental health of adolescents living in border areas. International Journal of Scientific and Research Publications. 2013; 3(1):1-4.

[41] Hooman H. [Modeling structural equations using LISREL software (Persian)]. Tehran: SAMT Publication; 2005.

[42] Kane GC, Gotto JL, West S, Hojat M, Mangione S. Jefferson scale of patient's perceptions of physician empathy: Preliminary psychometric data. Croatian Medical Journal. 2007; 48(1):81-6.

[43] Faraji S. [The relation between anxiety and depression, perception of the desease, and physician empathy in patients with arrhythmias, heart disease (Persian)] [MSc. thesis]. Tehran: Tehran Psychiatric Institute; 2008.
[44] Leahy RL. A model of emotional schemas. Cognitive and Behavioral Practice. 2002; 9(3):177-90. doi: 10.1016/s10777229(02)80048-7

[45] Leahy RL, Tirch D, Napolitano LA. Emotion regulation in psychotherapy: A practitioner's guide. New York: Guilford Press; 2011.

[46] Connor KM, Davidson JRT. Development of a new resilience scale: The Connor-Davidson resilience scale (CD-RISC). Depression and Anxiety. 2003; 18(2):76-82. doi: 10.1002/da.10113

[47] Mears GS. Examining the relationship between emotional schemas, emotional intelligence, and relationship satisfaction $[\mathrm{PhD}$ dissertation]. Lynchburg: Liberty University; 2012.

[48] Carle AC, Chassin L. Resilience in a community sample of children of alcoholics: Its prevalence and relation to internalizing symptomatology and positive affect. Journal of Applied Developmental Psychology. 2004; 25(5):577-95. doi: 10.1016/j.appdev.2004.08.005

[49] Tugade MM, Fredrickson BL. Resilient individuals use positive emotions to bounce back from negative emotional experiences. Journal of Personality and Social Psychology. 2004; 86(2):320-33. doi: $10.1037 / 0022-3514.86 .2 .320$

[50] Dent M. Really surviving teaching: Real resilience and passion. Educating Young Children: Learning and Teaching in the Early Childhood Years. 2013; 19(2):27.

[51] Hosini Ghomi T, Salimi Begestani H. [Effectiveness of resilience training on stress of mothers whose children, who were suffer from cancer in Imam Khomeini hospital of Tehran (Persian)]. Journal of Health Psychology. 2013; 1(4):97-109. 
